\title{
The utility of EEG spectral analysis in epileptiform activity identification in a child under general anesthesia
}

Liliane Godinho1; Marta Carvalho2; Jacinta Sá2; Pedro Amorim²

1- Centro Hospitalar Universitário de Coimbra | 2- Centro Hospitalar do Porto

\section{INTRODUCTION}

- In a patient under general anesthesia, epileptiform discharges may only be diagnosed if an electroencephalogram is performed.

- Intraoperative monitoring with bilateral BIS may be used to diagnose epileptiform activity in this setting, namely spectral analysis.

- We report a case in which bilateral BIS allowed for the diagnosis of epileptiform discharges.

\section{CASE REPORT}

A 12-year-old girl was submitted to surgical correction of a lumbar scoliosis through posterior approach.

Past medical history: neuromuscular scoliosis, spastic quadriplegic cerebral palsy (post-anoxic)

$\square 5^{\text {th }}$ grade Elementary School, Special Educations Needs. Speech Therapy Programme, Occupational Therapy, Hydrotherapy.

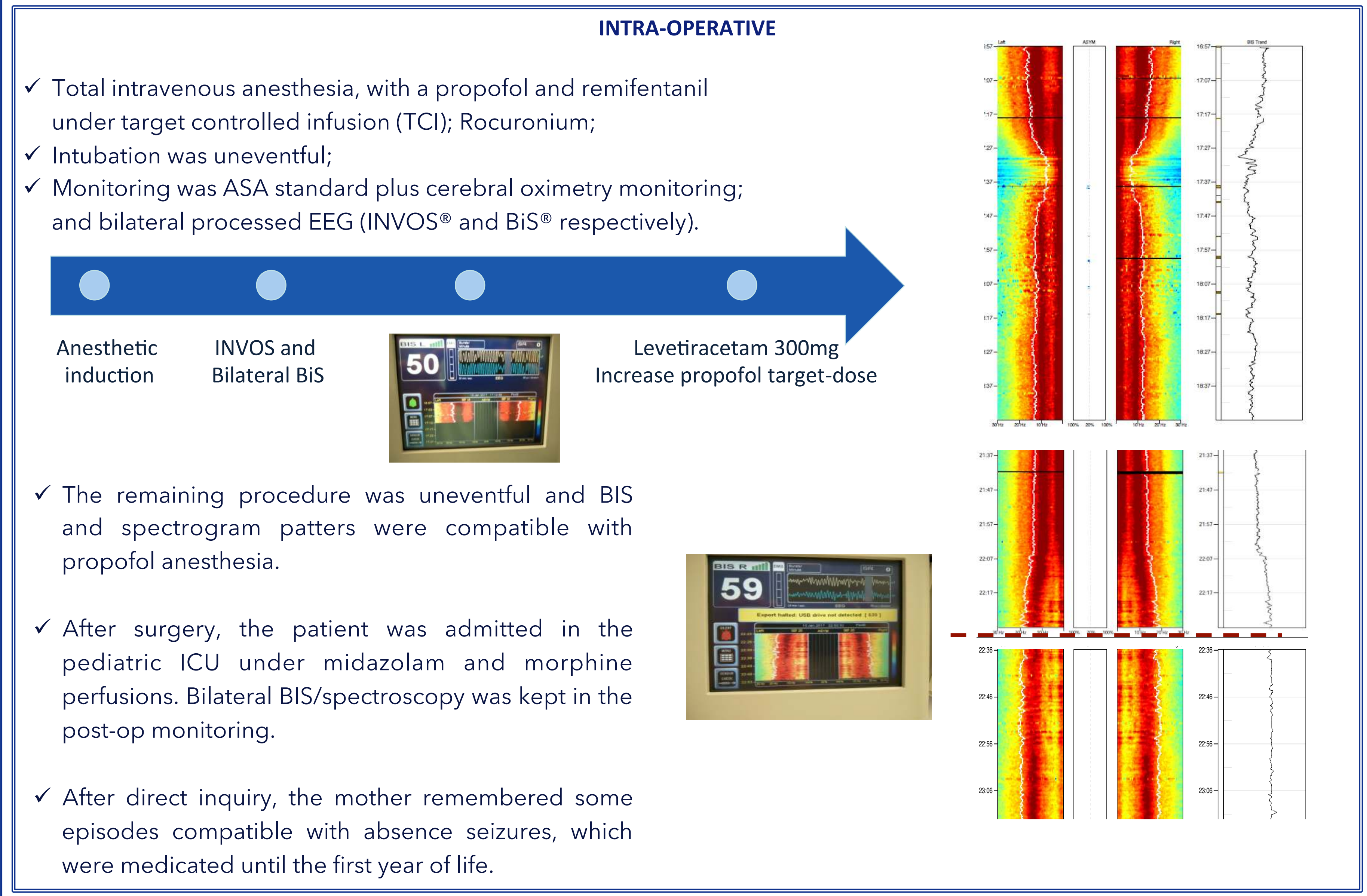

\section{DISCUSSION}

- Bilateral BIS spectroscopy may allow for the recognition of epileptiform discharges, but confirmation with conventional EEG is necessary.

- There seems to be a good correlation between spectroscopy patterns and the utilized drugs and concentrations, allowing for a better control of the anesthetic process.

- The use of bilateral BIS in the ICU setting may detect epileptiform activity and impact clinical and therapeutic decisions.

\section{LEARNING POINTS}

$\checkmark$ Spectrogram monitoring with bilateral BIS may be used as a supplementary tool to detect epileptiform activity, especially when continuous EEG monitoring is not available.

The definite diagnosis of epileptiform activity may only be established with an conventional EEG 